\title{
A Polynomial Blossom for the Askey-Wilson Operator
}

\author{
Plamen Simeonov ${ }^{1}$ - Ron Goldman ${ }^{2}$
}

Received: 4 September 2017 / Revised: 10 April 2018 / Accepted: 3 May 2018

C Springer Science+Business Media, LLC, part of Springer Nature 2018

\begin{abstract}
We introduce a blossoming procedure for polynomials related to the Askey-Wilson operator. This new blossom is symmetric, multiaffine, and reduces to the complex representation of the polynomial on a certain diagonal. This AskeyWilson blossom can be used to find the Askey-Wilson derivative of a polynomial of any order. We also introduce a corresponding Askey-Wilson Bernstein basis for which this new blossom provides the dual functionals. We derive a partition of unity property and a Marsden identity for this Askey-Wilson Bernstein basis, which turn out to be the terminating versions of Rogers' ${ }_{6} \phi_{5}$ summation formula and a very-well-poised ${ }_{8} \phi_{7}$ summation formula. Recurrence and symmetry relations and differentiation and degree elevation formulas for the Askey-Wilson Bernstein bases, as well as degree elevation formulas for Askey-Wilson Bézier curves, are also given.
\end{abstract}

Keywords Askey-Wilson operator · polynomial blossom - Bernstein basis · Bézier curve $\cdot$ dual functional property $\cdot$ Marsden identity $\cdot$ basic hypergeometric series . degree elevation

Mathematics Subject Classification Primary 33Dxx · 47B39 - 65D17 · 30C10; Secondary 41Axx

Communicated by Mourad Ismail.

Plamen Simeonov

simeonovp@uhd.edu

Ron Goldman

rng@cs.rice.edu

1 Department of Mathematics and Statistics, University of Houston-Downtown, Houston, TX 77002, USA

2 Department of Computer Science, Rice University, Houston, TX 77251, USA 


\section{Introduction}

Blossoming, Bernstein bases, and B-splines lie at the core of computer-aided geometric design [4,5,16-18]. Blossoming in particular is a powerful and efficient tool for analyzing Bézier and B-spline curves and surfaces [4,5]. Formulas for subdivision, knot insertion, differentiation, and degree elevation can all be readily derived from blossoming [4,5]. There are also other forms of the polynomial blossom: quantum blossoms, adapted to quantum derivatives $[6,21,22]$ and quantum splines-piecewise polynomials whose quantum derivatives agree at the joins $[8,20]$. Blossoming has also been extended to Müntz spaces [2], to trigonometric splines [11,14], and to Chebyshev spaces $[9,12,13]$.

For polynomials of degree $n$, the classical blossom and its quantum analogues are uniquely defined by three axioms: they are symmetric, multiaffine functions in $n$ variables that reduce to the original polynomial by substituting for these variables certain univariate linear functions. This last property is called the diagonal property of the blossom; different diagonal properties lead to different blossoms. In addition, these polynomial blossoms have multilinear analogues that can be used to compute classical derivatives (using the classical blossom) [4] or quantum derivatives (using quantum blossoms) $[6,8,20]$ of polynomials. This relationship appears to be common among degree lowering operators such as derivatives and appropriately defined polynomial blossoms. One such operator is the Askey-Wilson divided difference operator, which plays a fundamental role in the theory of orthogonal polynomials, basic hypergeometric series, and special functions $[1,3,10,15]$.

One of the main goals of this paper is to introduce blossoming, which has been so successful in the field of computer-aided geometric design, to the field of hypergeometric series. We shall define a symmetric and multiaffine polynomial blossom with an appropriate diagonal property so that its multilinear analogue can be used to evaluate Askey-Wilson derivatives of polynomials. We shall also explore the standard properties that accompany polynomial blossoms such as recursive evaluation algorithms, Askey-Wilson Bernstein bases, and various identities for these Askey-Wilson Bernstein bases. We will show that some of the standard identities such as the partition of unity property and the Marsden identity for the Askey-Wilson Bernstein bases reduce to summation formulas for basic hypergeometric series: Rogers' ${ }_{6} \phi_{5}$ summation formula and a summation formula for a very-well-poised ${ }_{8} \phi_{7}$ series. Thus we will extend the blossoming theory to the Askey-Wilson divided difference operator, establishing a natural connection between two previously unrelated subjects: polynomial blossoming and basic hypergeometric series.

We proceed in the following fashion. In Sect. 2 we establish our notation, and in Sect. 3 we review the Askey-Wilson divided difference operator. We define and prove the existence and uniqueness of the Askey-Wilson blossom in Sect. 4. We also show that the multilinear version of this Askey-Wilson blossom can be used to evaluate Askey-Wilson derivatives of polynomials. Sect. 5 is devoted to deriving recursive evaluation algorithms for the Askey-Wilson blossom. We apply these recursive evaluation algorithms in Sect. 6 to introduce the Askey-Wilson analogues of the Bernstein basis functions and to derive recurrence relations and explicit formulas for these Askey-Wilson Bernstein basis functions. We also derive a pair of symmetry relations, 
a pair of two-term differentiation formulas, and a two-term degree elevation formula for these functions. In Sect. 7 we derive identities for the Askey-Wilson Bernstein bases, and we use these identities to provide new straightforward blossoming proofs of two basic hypergeometric sums. A formula for the control points of degree elevated Askey-Wilson Bézier curves is also given in this section.

\section{Notation}

Throughout this paper we shall use the following standard notation from the theory of basic hypergeometric series $[1,3,10,15]$. The $q$-integers are defined by

$$
[0]_{q}=1, \quad[n]_{q}=\left\{\begin{array}{lll}
\left(q^{n}-1\right) /(q-1), & q \neq 1 \\
n, & q=1,
\end{array} \quad n \in \mathbb{N}\right.
$$

the $q$-shifted factorials are defined by

$$
[0]_{q} !=1, \quad[n]_{q} !=[1]_{q} \cdots[n]_{q}, \quad n \in \mathbb{N}
$$

and the $q$-binomial coefficients are defined by

$$
\left[\begin{array}{l}
n \\
k
\end{array}\right]_{q}=\frac{[n]_{q} !}{[k]_{q} ![n-k]_{q} !}, \quad k=0, \ldots, n
$$

We shall also use the notation $(a ; q)_{0}=1$,

$$
\begin{aligned}
(a ; q)_{n} & =\prod_{k=0}^{n-1}\left(1-q^{k} a\right), \quad n \in \mathbb{N} \cup\{\infty\}, \quad\left(a_{1}, \ldots, a_{m} ; q\right)_{n}=\prod_{j=1}^{m}\left(a_{j} ; q\right)_{n}, \\
n & \in \mathbb{N}_{0} \cup\{\infty\} .
\end{aligned}
$$

$\mathrm{A}{ }_{r} \phi_{s}$ basic hypergeometric series is defined by [10, (12.1.6)]

$$
{ }_{r} \phi_{s}\left(\begin{array}{c}
a_{1}, \ldots, a_{r} \\
b_{1}, \ldots, b_{s}
\end{array} \mid q, z\right)=\sum_{k=0}^{\infty} \frac{\left(a_{1}, \ldots, a_{r} ; q\right)_{k}}{\left(q, b_{1}, \ldots, b_{s} ; q\right)_{k}}\left(-q^{(k-1) / 2}\right)^{(s+1-r) k} z^{k}
$$

A very-well-poised ${ }_{r+1} W_{r}$ hypergeometric series is defined by [10, (12.5.12)]

$$
{ }_{r+3} W_{r+2}\left(\alpha ; \alpha_{1}, \ldots, \alpha_{r} ; z\right)={ }_{r+3} \phi_{r+2}\left(\begin{array}{c}
\alpha, q \sqrt{\alpha},-q \sqrt{\alpha}, \alpha_{1}, \ldots, \alpha_{r} \\
\sqrt{\alpha},-\sqrt{\alpha}, q \alpha / \alpha_{1}, \ldots, q \alpha / \alpha_{r}
\end{array} \mid q, z\right)
$$

Throughout this paper, $\mathbb{N}$ denotes the set of positive integers, $\mathbb{N}_{0}=\mathbb{N} \cup\{0\}$, and for all infinite products and series involving $q$, it is assumed that $|q|<1$. 


\section{The Askey-Wilson Operator}

To define the Askey-Wilson operator, we begin by setting $x=\cos \theta$ and $f(x)=$ $\breve{f}\left(e^{i \theta}\right)$, where

$$
\breve{f}(z)=f(\varphi(z)), \quad \varphi(z)=(z+1 / z) / 2, \quad z=e^{ \pm i \theta} .
$$

Here $\theta$ may be real or complex, $x=\varphi(z)$, and $z=x+\sqrt{x^{2}-1}$, using the branch of the square root for which $\sqrt{x}>0$ for $x>0$.

Fix $q \in(0,1)$. The Askey-Wilson divided difference operator or the Askey-Wilson operator or the Askey-Wilson derivative $\mathcal{D}_{q}$ is defined by [10, (12.1.10)], [15]

$$
\left(\mathcal{D}_{q} f\right)(x)=\left(\mathcal{D}_{q} \breve{f}\right)(z)=\frac{\breve{f}\left(q^{1 / 2} z\right)-\breve{f}\left(q^{-1 / 2} z\right)}{\varphi\left(q^{1 / 2} z\right)-\varphi\left(q^{-1 / 2} z\right)}=\frac{f\left(\varphi\left(q^{1 / 2} z\right)\right)-f\left(\varphi\left(q^{-1 / 2} z\right)\right)}{\varphi\left(q^{1 / 2} z\right)-\varphi\left(q^{-1 / 2} z\right)},
$$

with $x=\varphi(z), z=e^{i \theta}$. Since $\varphi(1 / z)=\varphi(z)$, it follows from (3.1) and (3.2) that $\breve{f}(1 / z)=\breve{f}(z)$ and $\left(\mathcal{D}_{q} \breve{f}\right)(1 / z)=\left(\mathcal{D}_{q} \breve{f}\right)(z)$.

Later in this paper, to simplify certain expressions, we shall use the following straightforward identities:

$$
\begin{aligned}
& \varphi(z)-\varphi(w)=(z-w)(1-1 /(z w)) / 2=-(z-w)(1 / z-w) /(2 w), \\
& \varphi(z)-\varphi(w)=-w(1-z / w)(1-1 /(z w)) / 2=-(1-w z)(1-w / z) /(2 w), \\
& \varphi(\lambda z)-\varphi\left(\lambda^{-1} z\right)=\left(\lambda-\lambda^{-1}\right)(z-1 / z) / 2 .
\end{aligned}
$$

\section{The Askey-Wilson Blossom}

We begin this section with a lemma and a corollary, which will be used to establish the existence and uniqueness of the Askey-Wilson blossom.

Lemma 4.1 Let $R(z)$ be a rational function of $z$ such that $R(1 / z)=R(z)$ and $z^{n} R(z)$ is a polynomial of degree $2 n$ in $z$. Then $R(z)$ is a polynomial of degree $n$ in $x=\varphi(z)$.

Proof Setting $z=e^{i \theta}$ and $x=\cos \theta$, we get $z^{k}+z^{-k}=2 \cos (k \theta)=2 T_{k}(x)$, where $T_{k}(x)$ is the degree $k$ Chebyshev polynomial of the first kind [10, (4.5.18)], [15]. It follows from the properties of $R(z)$ that $R(z)=\sum_{k=0}^{n} r_{k}\left(z^{k}+z^{-k}\right)=2 \sum_{k=0}^{n} r_{k} T_{k}(x)$.

Corollary 4.2 The operator $\mathcal{D}_{q}$ acts on the polynomials as a degree lowering operator.

Proof By (3.2) we have

$$
\mathcal{D}_{q}\left(x^{n}\right)=\mathcal{D}_{q}\left(\varphi(z)^{n}\right)=\sum_{k=0}^{n-1} \varphi\left(q^{1 / 2} z\right)^{k} \varphi\left(q^{-1 / 2} z\right)^{n-1-k}:=R_{n-1}(z)
$$


Since $\varphi(z)=\varphi(1 / z)$, it follows from (4.1) that $R_{n-1}(1 / z)=R_{n-1}(z)$. Therefore by (4.1) and Lemma $4.1, R_{n-1}(z)$ is a degree $n-1$ polynomial in $x=\varphi(z)$.

Definition 4.3 The Askey-Wilson blossom or AW-blossom of a degree $n$ polynomial $P(x)$ is the unique symmetric multiaffine function $\breve{p}\left(u_{1}, \ldots, u_{n}\right)$ such that

$$
\breve{p}\left(\varphi\left(q^{-(n-1) / 2} z\right), \varphi\left(q^{1-(n-1) / 2} z\right), \ldots, \varphi\left(q^{(n-1) / 2} z\right)\right)=\breve{P}(z) .
$$

Theorem 4.4 Every polynomial of degree $n$ has a unique $A W$-blossom $\breve{p}\left(u_{1}, \ldots, u_{n}\right)$.

Proof First we define the functions

$$
F_{n, k}(x ; q)=e_{n, k}\left(\varphi\left(q^{-(n-1) / 2} z\right), \varphi\left(q^{1-(n-1) / 2} z\right), \ldots, \varphi\left(q^{(n-1) / 2} z\right)\right), \quad k=0, \ldots, n,
$$

where $x=\varphi(z)$, and $e_{n, 0}\left(u_{1}, \ldots, u_{n}\right)=1$ and

$$
e_{n, k}\left(u_{1}, \ldots, u_{n}\right)=\sum_{1 \leq j_{1}<\cdots<j_{k} \leq n} \prod_{\nu=1}^{k} u_{j_{v}}, \quad k=1, \ldots, n,
$$

are the elementary symmetric functions in $n$ variables. Let $R_{n, k}(z ; q)$ denote the righthand side of (4.3). Then $R_{n, k}(1 / z ; q)=R_{n, k}(z ; q)$, and $z^{k} R_{n, k}(z ; q)$ is a polynomial in $z$ of exact degree $2 k$. By Lemma $4.1, R_{n, k}(z ; q)$ is a polynomial in $x=\varphi(z)$ of exact degree $k$ for each $k=0, \ldots, n$. Thus $\left\{F_{n, k}(x ; q)\right\}_{k=0}^{n}$ is a basis for the degree $n$ polynomials in $x$.

Let $P(x)$ be a degree $n$ polynomial. Then $P(x)=\sum_{k=0}^{n} a_{k}(q) F_{n, k}(x ; q)$. Consider the function

$$
\breve{p}\left(u_{1}, \ldots, u_{n}\right)=\sum_{k=0}^{n} a_{k}(q) e_{n, k}\left(u_{1}, \ldots, u_{n}\right) .
$$

Clearly $\breve{p}\left(u_{1}, \ldots, u_{n}\right)$ is symmetric and multiaffine, and setting $u_{j}=\varphi\left(q^{j-1-(n-1) / 2}\right.$ $z), j=1, \ldots, n$ in $\breve{p}\left(u_{1}, \ldots, u_{n}\right)$ yields $P(x)$. Therefore $\breve{p}\left(u_{1}, \ldots, u_{n}\right)$ is an AWblossom of $P(x)$.

The proof of uniqueness is standard. Assume that $\breve{p}_{1}\left(u_{1}, \ldots, u_{n}\right)$ and $\breve{p}_{2}\left(u_{1}, \ldots\right.$, $u_{n}$ ) are two AW-blossoms of $P(x)$. Since every symmetric and multiaffine function of $\left\{u_{j}\right\}_{j=1}^{n}$ has a unique representation as a linear combination of the elementary symmetric functions of $\left\{u_{j}\right\}_{j=1}^{n}$,

$$
\breve{p}_{v}\left(u_{1}, \ldots, u_{n}\right)=\sum_{k=0}^{n} b_{v, k} e_{n, k}\left(u_{1}, \ldots, u_{n}\right), \quad v=1,2
$$

Setting $u_{j}=\varphi\left(q^{j-1-(n-1) / 2} z\right), j=1, \ldots, n$ in the last equation and using the diagonal property of the AW-blossom yields $\sum_{k=0}^{n} b_{1, k} F_{n, k}(x ; q)=P(x)=$ $\sum_{k=0}^{n} b_{2, k} F_{n, k}(x ; q)$. Since the polynomials $\left\{F_{n, k}(x ; q)\right\}_{k=0}^{n}$ are linearly independent, it follows that $b_{1, k}=b_{2, k}, k=0, \ldots, n$. 
The AW-blossoms of the monomials $\left\{x^{k}\right\}_{k=1}^{n}$ are, in general, hard to compute, since the monomials are not easy to express in terms of the natural polynomial basis $\left\{F_{n, k}(x ; q)\right\}_{k=0}^{n}$ for the AW-blossom. The polynomials $\left\{F_{n, k}(x ; q)\right\}_{k=0}^{n}$ can be expressed recursively in terms of the monomials using the Newton-Girard identities [23], and then the corresponding triangular coefficient matrix can be inverted. As an example, here we compute the AW-blossom of $x$. By (4.3),

$$
\begin{aligned}
F_{n, 1}(x ; q) & =\sum_{j=0}^{n-1} \varphi\left(q^{j-(n-1) / 2} z\right)=\left(q^{-(n-1) / 2}[n]_{q} z+q^{(n-1) / 2}[n]_{1 / q} / z\right) / 2 \\
& =q^{-(n-1) / 2}[n]_{q} x .
\end{aligned}
$$

Therefore,

$$
\breve{x}\left(u_{1}, \ldots, u_{n}\right)=q^{(n-1) / 2} e_{n, 1}\left(u_{1}, \ldots, u_{n}\right) /[n]_{q} .
$$

The homogeneous $A W$-blossom of a degree $n$ polynomial $P(x)$ is the homogenization of the AW-blossom $\breve{p}\left(u_{1}, \ldots, u_{n}\right)$ of $P(x)$, defined by

$$
\breve{p}\left(\left(u_{1}, w_{1}\right), \ldots,\left(u_{n}, w_{n}\right)\right)=w_{1} \cdots w_{n} \breve{p}\left(u_{1} / w_{1}, \ldots, u_{n} / w_{n}\right) .
$$

In Theorem 4.4 we proved that the AW-blossom of $P(x)$ is given by (4.5). Combining (4.8) and (4.5) yields a formula for the homogeneous AW-blossom of $P(x)$.

By definition, the homogeneous AW-blossom of $P(x)$ is a symmetric and multilinear function in the pairs $\left\{\left(u_{j}, w_{j}\right)\right\}_{j=1}^{n}$ and has the diagonal property

$$
\breve{p}\left(\left(\varphi\left(q^{-(n-1) / 2} z\right), 1\right), \ldots,\left(\varphi\left(q^{(n-1) / 2} z\right), 1\right)\right)=\breve{P}(z)
$$

Next we will show that the homogeneous AW-blossom can be used to find AWderivatives of polynomials.

Proposition 4.5 Let $P(x)$ be a polynomial of degree $n$, and let $\breve{p}\left(\left(u_{1}, w_{1}\right), \ldots\right.$, $\left.\left(u_{n}, w_{n}\right)\right)$ be the homogeneous AW-blossom of $P(x)$. Set $\delta=(1,0)$ and $\delta^{[m]}=$ $\underbrace{\delta, \ldots, \delta}_{m}$. Then

$$
\left(\mathcal{D}_{q}^{m} \breve{P}\right)(z)=\kappa_{n, m}(q) \breve{p}\left(\left(\varphi\left(q^{-(n-1-m) / 2} z\right), 1\right), \ldots,\left(\varphi\left(q^{(n-1-m) / 2} z\right), 1\right), \delta^{[m]}\right)
$$

$m=0, \ldots, n$, where

$$
\kappa_{n, m}(q)=q^{-\frac{1}{2}\left(m n-\left(\begin{array}{c}
m+1 \\
2
\end{array}\right)\right)}[n]_{q} ! /[n-m]_{q} !, \quad m=0, \ldots, n .
$$


Proof The case $m=0$ of (4.10)-(4.11) is (4.9). To prove this result for $m=1$, observe that by (4.9), the symmetry and multilinearity of the homogeneous AW-blossom, and (3.5), it follows that

$$
\begin{aligned}
& \left(\mathcal{D}_{q} \breve{P}\right)(z)=\frac{\breve{P}\left(q^{1 / 2} z\right)-\breve{P}\left(q^{-1 / 2} z\right)}{\varphi\left(q^{1 / 2} z\right)-\varphi\left(q^{-1 / 2} z\right)} \\
& =\frac{\breve{p}\left(\left(\varphi\left(q^{-(n-2) / 2} z\right), 1\right), \ldots,\left(\varphi\left(q^{n / 2} z\right), 1\right)\right)-\breve{p}\left(\left(\varphi\left(q^{-n / 2} z\right), 1\right), \ldots,\left(\varphi\left(q^{(n-2) / 2} z\right), 1\right)\right)}{\varphi\left(q^{1 / 2} z\right)-\varphi\left(q^{-1 / 2} z\right)} \\
& =\breve{p}\left(\left(\varphi\left(q^{-(n-2) / 2} z\right), 1\right), \ldots,\left(\varphi\left(q^{(n-2) / 2} z\right), 1\right),\left(\frac{\varphi\left(q^{n / 2} z\right)-\varphi\left(q^{-n / 2} z\right)}{\varphi\left(q^{1 / 2} z\right)-\varphi\left(q^{-1 / 2} z\right)}, 0\right)\right) \\
& =q^{-(n-1) / 2}[n]_{q} \breve{p}\left(\left(\varphi\left(q^{-(n-2) / 2} z\right), 1\right), \ldots,\left(\varphi\left(q^{(n-2) / 2} z\right), 1\right), \delta\right) .
\end{aligned}
$$

We now proceed by induction on $m$. Assume that (4.10)-(4.11) holds for some $m<n$. Notice that

$$
\kappa_{n, m}(q) \breve{p}\left(\left(u_{1}, w_{1}\right), \ldots,\left(u_{n-m}, w_{n-m}\right), \delta^{[m]}\right)
$$

is a symmetric multilinear function of $\left\{\left(u_{j}, w_{j}\right)\right\}_{j=1}^{n-m}$, which for $\left(u_{j}, w_{j}\right)=$ $\left(\varphi\left(q^{j-1-(n-1-m) / 2} z\right), 1\right), j=1, \ldots, n-m$, reduces to $\left(\mathcal{D}_{q}^{m} \breve{P}\right)(z)$ by (4.10). Hence the function in (4.13) is the homogeneous AW-blossom of $\left(\mathcal{D}_{q}^{m} \breve{P}\right)(z)$. Therefore,

$$
\begin{aligned}
\frac{\left(\mathcal{D}_{q}^{m+1} \breve{P}\right)(z)}{\kappa_{n, m}(q)}= & \frac{\mathcal{D}_{q}\left(\left(\mathcal{D}_{q}^{m} \breve{P}\right)(z)\right)}{\kappa_{n, m}(q)}=\frac{\left(\mathcal{D}_{q}^{m} \breve{P}\right)\left(q^{1 / 2} z\right)-\left(\mathcal{D}_{q}^{m} \breve{P}\right)\left(q^{-1 / 2} z\right)}{\kappa_{n, m}(q)\left(\varphi\left(q^{1 / 2} z\right)-\varphi\left(q^{-1 / 2} z\right)\right)} \\
= & \frac{1}{\varphi\left(q^{1 / 2} z\right)-\varphi\left(q^{-1 / 2} z\right)}\left\{\breve{p}\left(\left(\varphi\left(q^{-(n-2-m) / 2} z\right), 1\right), \ldots,\left(\varphi\left(q^{(n-m) / 2} z\right), 1\right), \delta^{[m]}\right)\right. \\
& \left.-\breve{p}\left(\left(\varphi\left(q^{-(n-m) / 2} z\right), 1\right), \ldots,\left(\varphi\left(q^{(n-2-m) / 2} z\right), 1\right), \delta^{[m]}\right)\right\} \\
= & \breve{p}\left(\left(\varphi\left(q^{-(n-2-m) / 2} z\right), 1\right), \ldots,\left(\varphi\left(q^{(n-2-m) / 2} z\right), 1\right),\left(\frac{\varphi\left(q^{(n-m) / 2} z\right)-\varphi\left(q^{-(n-m) / 2} z\right)}{\varphi\left(q^{1 / 2} z\right)-\varphi\left(q^{-1 / 2} z\right)}, 0\right), \delta^{[m]}\right) \\
= & q^{-(n-m-1) / 2}[n-m]_{q} \breve{p}\left(\left(\varphi\left(q^{-(n-2-m) / 2} z\right), 1\right), \ldots,\left(\varphi\left(q^{(n-2-m) / 2} z\right), 1\right), \delta^{[m+1]}\right),
\end{aligned}
$$

where we used the diagonal, symmetry, and multilinear properties of the homogeneous AW-blossom of $\left(\mathcal{D}_{q}^{m} P\right)(z)$, along with (3.5). Multiplying (4.14) by $\kappa_{n, m}(q)$ yields (4.10)-(4.11) for the case $m+1$, since by (4.11) we have $q^{-(n-m-1) / 2}[n-$ $m]_{q} \kappa_{n, m}(q)=\kappa_{n, m+1}(q)$.

As an example, we next compute the AW-derivatives of the polynomials $\left\{F_{n, k}(x ; q)\right\}$ defined by (4.3). The multilinear AW-blossom of $F_{n, k}(x ; q)$ is $w_{1} \cdots w_{n} e_{n, k}\left(u_{1} / w_{1}, \ldots, u_{n} / w_{n}\right)$, which reduces to $w_{1} \cdots w_{n-1} e_{n-1, k-1}\left(u_{1} / w_{1}\right.$, $\left.\ldots, u_{n-1} / w_{n-1}\right)$ when $\left(u_{n}, w_{n}\right)=\delta$. By Proposition 4.5 and (4.3),

$$
\mathcal{D}_{q} \breve{F}_{n, k}(z ; q)=q^{-(n-1) / 2}[n]_{q} \breve{F}_{n-1, k-1}(z ; q) .
$$

Iterating this equation yields

$$
\mathcal{D}_{q}^{m} \breve{F}_{n, k}(z ; q)=\kappa_{n, m}(q) \breve{F}_{n-m, k-m}(z ; q), \quad m \leq k \leq n,
$$

and 0 if $m>k$. 


\section{Recursive Evaluation Algorithms}

In this section we will give recursive evaluation algorithms for the AW-blossom and for polynomials and their AW-derivatives in terms of their AW-blossom.

We shall call a sequence $\left(\alpha_{0}, \ldots, \alpha_{n-1}, \beta_{0}, \ldots, \beta_{n-1}\right)$ of real or complex numbers a progressive parameter sequence if $\beta_{l} \neq \alpha_{k}, 0 \leq l \leq k \leq n-1$.

We shall also call a sequence $\left(\left(\alpha_{0}, \xi_{0}\right), \ldots,\left(\alpha_{n-1}, \xi_{n-1}\right),\left(\beta_{0}, \eta_{0}\right),\left(\beta_{n-1}, \eta_{n-1}\right)\right)$ of pairs of real or complex numbers a progressive parameter sequence if $\beta_{l} \xi_{k} \neq \alpha_{k} \eta_{l}$, $0 \leq l \leq k \leq n-1$.

Proposition 5.1 (Recursive Evaluation for the Askey-Wilson Blossom) Let $P(x)$ be a degree $n$ polynomial. The recursive evaluation algorithm for the AW-blossom $\breve{p}\left(\left(u_{1}, w_{1}\right), \ldots,\left(u_{n}, w_{n}\right)\right)$ of $\breve{P}(z)$ with respect to the progressive parameter sequence $\left(\left(\alpha_{0}, \xi_{0}\right), \ldots,\left(\alpha_{n-1}, \xi_{n-1}\right),\left(\beta_{0}, \eta_{0}\right), \ldots,\left(\beta_{n-1}, \eta_{n-1}\right)\right)$ is generated as follows: Set

$$
Q_{k}^{0}=\breve{p}\left(\left(\alpha_{k}, \xi_{k}\right), \ldots,\left(\alpha_{n-1}, \xi_{n-1}\right),\left(\beta_{0}, \eta_{0}\right), \ldots,\left(\beta_{k-1}, \eta_{k-1}\right)\right), \quad k=0, \ldots, n,
$$

and define recursively

$$
Q_{k}^{s}=\frac{\beta_{k} w_{s}-\eta_{k} u_{s}}{\beta_{k} \xi_{k+s-1}-\alpha_{k+s-1} \eta_{k}} Q_{k}^{s-1}+\frac{\xi_{k+s-1} u_{s}-\alpha_{k+s-1} w_{s}}{\beta_{k} \xi_{k+s-1}-\alpha_{k+s-1} \eta_{k}} Q_{k+1}^{s-1},
$$

$k=0, \ldots, n-s, s=1, \ldots, n$. Then

$$
Q_{k}^{s}=\breve{p}\left(\left(u_{1}, w_{1}\right), \ldots,\left(u_{s}, w_{s}\right),\left(\alpha_{k+s}, \xi_{k+s}\right), \ldots,\left(\alpha_{n-1}, \xi_{n-1}\right),\left(\beta_{0}, \eta_{0}\right), \ldots,\left(\beta_{k-1}, \eta_{k-1}\right)\right),
$$

$k=0, \ldots, n-s, s=1, \ldots, n$. In particular

$$
Q_{0}^{n}=\breve{p}\left(\left(u_{1}, w_{1}\right), \ldots,\left(u_{n}, w_{n}\right)\right) .
$$

Proof This result follows directly from the symmetry and multiaffine properties of the AW-blossom, by induction on $s$.

Let $S_{n}$ denote the set of all permutations of the numbers $\{1, \ldots, n\}$.

Corollary 5.2 (Recursive Evaluation for Polynomials) Let $P(x)$ be a degree $n$ polynomial with homogeneous $A W$-blossom $\breve{p}\left(\left(u_{1}, w_{1}\right), \ldots,\left(u_{n}, w_{n}\right)\right)$. A recursive evaluation algorithm for $\breve{P}(z)$ with respect to a progressive sequence $\left(\left(\alpha_{0}, \xi_{0}\right), \ldots\right.$, $\left.\left(\alpha_{n-1}, \xi_{n-1}\right),\left(\beta_{0}, \eta_{0}\right), \ldots,\left(\beta_{n-1}, \eta_{n-1}\right)\right)$ and a permutation $\sigma \in S_{n}$ is generated as follows: Set

$\breve{P}_{k}^{0}(z)=\breve{p}\left(\left(\alpha_{k}, \xi_{k}\right), \ldots,\left(\alpha_{n-1}, \xi_{n-1}\right),\left(\beta_{0}, \eta_{0}\right), \ldots,\left(\beta_{k-1}, \eta_{k-1}\right)\right), \quad k=0, \ldots, n$ 
and with $\xi=q^{-(n-1) / 2} z$ define recursively

$$
\breve{P}_{k}^{s}(z)=\frac{\beta_{k}-\eta_{k} \varphi\left(q^{\sigma(s)-1} \xi\right)}{\beta_{k} \xi_{k+s-1}-\alpha_{k+s-1} \eta_{k}} \breve{P}_{k}^{s-1}(z)+\frac{\xi_{k+s-1} \varphi\left(q^{\sigma(s)-1} \xi\right)-\alpha_{k+s-1}}{\beta_{k} \xi_{k+s-1}-\alpha_{k+s-1} \eta_{k}} \breve{P}_{k+1}^{s-1}(z),
$$

$k=0, \ldots, n-s, s=1, \ldots, n$. Then

$$
\begin{aligned}
\breve{P}_{k}^{s}(z)=\breve{p}( & \left(\varphi\left(q^{\sigma(1)-1} \xi\right), 1\right), \ldots,\left(\varphi\left(q^{\sigma(s)-1} \xi\right), 1\right) \\
& \left.\left(\alpha_{k+s}, \xi_{k+s}\right), \ldots,\left(\alpha_{n-1}, \xi_{n-1}\right),\left(\beta_{0}, \eta_{0}\right), \ldots,\left(\beta_{k-1}, \eta_{k-1}\right)\right)
\end{aligned}
$$

$k=0, \ldots, n-s, s=1, \ldots, n$. In particular

$$
\breve{P}_{0}^{n}(z)=\breve{p}\left(\left(\varphi\left(q^{\sigma(1)-1} \xi\right), 1\right), \ldots,\left(\varphi\left(q^{\sigma(n)-1} \xi\right), 1\right)\right)=\breve{P}(z)
$$

Proof Equations (5.6) follow from the symmetry and multiaffine properties of the AW-blossom, by induction on $s$. Equation (5.7) follows from the case $s=n$ of (5.6) and the symmetry and diagonal properties of the AW-blossom of $P(x)$.

Corollary 5.3 Let $P(x)$ be a degree $n$ polynomial, and let $1 \leq m \leq n$. Then for each subset $J=\left\{j_{\nu}\right\}_{\nu=1}^{n-m} \subset\{1, \ldots, n\}$ and each permutation $\sigma \in S_{n-m}$, the recursive evaluation algorithm of Proposition 5.1 with $\left(u_{j_{v}}, w_{j_{v}}\right)=\left(\varphi\left(q^{\sigma(v)-1} \xi\right), 1\right)$, $v=1, \ldots, n-m$, where $\xi=q^{-(n-m-1) / 2} z$, and $\left(u_{j}, w_{j}\right)=\delta, j \notin J$, yields $\kappa_{n, m}(q)^{-1}\left(\mathcal{D}_{q}^{m} \breve{P}\right)(z)$. There are $n ! / m$ ! such algorithms.

Proof This result follows from the symmetry of the AW-blossom and Proposition 4.5.

\section{The Askey-Wilson Bernstein Basis Functions}

In this section we will introduce the Askey-Wilson Bernstein basis, along with a dual functional property for the Askey-Wilson blossom relative to this Askey-Wilson Bernstein basis. We will also derive recursive relations and explicit formulas for the Askey-Wilson Bernstein basis functions.

Definition 6.1 Let $a, b \in \mathbb{C}$ be such that ${ }^{1}$

$$
\alpha_{l}=\varphi\left(q^{l-(n-1) / 2} a\right), \quad \beta_{l}=\varphi\left(q^{l-(n-1) / 2} b\right), \quad l=0, \ldots, n-1
$$

satisfy $\beta_{l} \neq \alpha_{k}, 0 \leq l \leq k \leq n-1$. For each $j=0, \ldots, n$, the $j$-th Askey-Wilson Bernstein basis function or $A W$-Bernstein basis function of degree $n$ with parameters $a$ and $b$ is the polynomial

$$
B_{j}^{n}(x ; a, b ; q)=\breve{B}_{j}^{n}(z ; a, b ; q)=\breve{P}_{0}^{n}(z), \quad x=\varphi(z)
$$

\footnotetext{
${ }^{1}$ For the classical Bernstein basis functions, these two parameters represent the end points of the domain interval, that is, the parameter range over which all the classical Bernstein basis functions are non-negative.
} 
generated by the recurrence (5.5) with $\xi_{l}=\eta_{l}=1, l=0, \ldots, n-1$, using the parameters $\left\{\alpha_{l}\right\}_{l=0}^{n-1}$ and $\left\{\beta_{l}\right\}_{l=0}^{n-1}$ defined by (6.1), and any permutation $\sigma \in S_{n}$, starting from the initial values $\breve{P}_{k}^{0}(z)=\delta_{k, j}, k=0, \ldots, n$. A polynomial curve of the form $P(x)=\sum_{j=0}^{n} P_{j} B_{j}^{n}(x ; a, b ; q)$ is called an Askey-Wilson Bézier curve of degree $n$ with parameters $a$ and $b$. The coefficients $\left\{P_{j}\right\}_{j=0}^{n}$ are called the Askey-Wilson Bézier control points or simply the control points of $P(x)$.

Proposition 6.2 (Dual Functional Property of the Askey-Wilson Blossom) Every polynomial $P(x)$ of degree $n$ has the representation

$$
P(x)=\breve{P}(z)=\sum_{j=0}^{n} \breve{p}\left(\alpha_{j}, \ldots, \alpha_{n-1}, \beta_{0}, \ldots, \beta_{j-1}\right) \breve{B}_{j}^{n}(z ; a, b ; q)
$$

Proof Equation (6.2) follows immediately from the definition of the degree $n$ AWBernstein basis functions, linearity, and Corollary 5.2.

Corollary 6.3 The Askey-Wilson Bernstein basis functions $\left\{\breve{B}_{j}^{n}(z ; a, b ; q)\right\}_{j=0}^{n}$ form a basis for the space of degree $n$ polynomials in $x=\varphi(z)$.

Next we derive two formulas for the AW-derivative of the AW-Bernstein basis functions.

Proposition 6.4 (Differentiation Formulas)

$$
\mathcal{D}_{q} \breve{B}_{j}^{n}(z ; a, b ; q)=2[n]_{q} \frac{\left\{\breve{B}_{j-1}^{n-1}\left(z ; q^{1 / 2} a, q^{1 / 2} b ; q\right)-\breve{B}_{j}^{n-1}\left(z ; q^{-1 / 2} a, q^{-1 / 2} b ; q\right)\right\}}{(1-1 /(a b))\left(b-q^{n-1} a\right)},
$$

$j=0, \ldots, n$, and

$\mathcal{D}_{q} \breve{B}_{j}^{n}(z ; a, b ; q)=\frac{2[n]_{q}}{b-a}\left\{\frac{\breve{B}_{j-1}^{n-1}\left(z ; q^{1 / 2} a, q^{-1 / 2} b ; q\right)}{q^{j-1}-q^{n-j} /(a b)}-\frac{\breve{B}_{j}^{n-1}\left(z ; q^{1 / 2} a, q^{-1 / 2} b ; q\right)}{q^{j}-q^{n-1-j} /(a b)}\right\}$, $j=0, \ldots, n$, where $\breve{B}_{-1}^{n-1}=\breve{B}_{n}^{n-1}=0$.

Proof Let $P(x)$ be a degree $n$ polynomial with multilinear AW-blossom $\breve{p}\left(\left(u_{1}, w_{1}\right)\right.$, $\left.\ldots,\left(u_{n}, w_{n}\right)\right)$. By Proposition $6.2, \breve{P}(z)=\sum_{k=0}^{n} P_{k} \breve{B}_{k}^{n}(z ; a, b ; q)$, where by $(6.2)$ and (4.8),

$$
P_{k}=\breve{p}\left(\left(\alpha_{k}, 1\right), \ldots,\left(\alpha_{n-1}, 1\right),\left(\beta_{0}, 1\right), \ldots,\left(\beta_{k-1}, 1\right)\right), \quad k=0, \ldots, n,
$$

with the parameter sequence defined by (6.1). Hence

$$
\left(\mathcal{D}_{q} \breve{P}\right)(z)=\sum_{k=0}^{n} P_{k} \mathcal{D}_{q} \breve{B}_{k}^{n}(z ; a, b ; q)
$$


On the other hand, by the case $m=1$ of Proposition 4.5,

$$
\left(\mathcal{D}_{q} \breve{P}\right)(z)=\kappa_{n, 1}(q) \breve{p}\left(\left(\varphi\left(q^{-(n-2) / 2} z\right), 1\right), \ldots,\left(\varphi\left(q^{(n-2) / 2} z\right), 1\right), \delta\right),
$$

with $\delta=(1,0)$. Equation $(6.7)$ shows that $\left(\mathcal{D}_{q} \breve{P}\right)(z)$ is a degree $n-1$ polynomial in $x=\varphi(z)$ with AW-blossom $\kappa_{n, 1}(q) \breve{p}\left(\left(u_{1}, 1\right), \ldots,\left(u_{n-1}, 1\right), \delta\right)$.

Now we prove (6.3). We evaluate $\left(\mathcal{D}_{q} \breve{P}\right)(z)$ using the recursive evaluation algorithm of Corollary 5.3 with $\left(u_{j}, w_{j}\right)=\left(\varphi\left(q^{j-1-(n-2) / 2} z\right), 1\right), j=1, \ldots, n-1,\left(u_{n}, w_{n}\right)=$ $\delta$, and parameter sequence $\left(\left(\alpha_{0}, 1\right), \ldots,\left(\alpha_{n-1}, 1\right),\left(\beta_{0}, 1\right), \ldots,\left(\beta_{n-1}, 1\right)\right)$. By (5.2) and (6.7), $\left(\mathcal{D}_{q} \breve{P}\right)(z)=\kappa_{n, 1}(q) \frac{Q_{1}^{n-1}-Q_{0}^{n-1}}{\beta_{0}-\alpha_{n-1}}$. Here $Q_{0}^{n-1}$ is a degree $n-1$ polynomial generated by the first $n-1$ steps of this algorithm from the control points $P_{0}, \ldots, P_{n-1}$ with parameter sequence $\left(\left(\alpha_{0}, 1\right), \ldots,\left(\alpha_{n-2}, 1\right),\left(\beta_{0}, 1\right), \ldots,\left(\beta_{n-2}, 1\right)\right)$; hence $Q_{0}^{n-1}$ $=\sum_{k=0}^{n-1} P_{k} \breve{B}_{k}^{n-1}\left(z ; q^{-1 / 2} a, q^{-1 / 2} b ; q\right)$. Similarly, $Q_{1}^{n-1}$ is a degree $n-1$ polynomial generated by the first $n-1$ steps of this algorithm from the control points $P_{1}, \ldots, P_{n}$ with parameter sequence $\left(\left(\alpha_{1}, 1\right), \ldots,\left(\alpha_{n-1}, 1\right),\left(\beta_{1}, 1\right), \ldots,\left(\beta_{n-1}, 1\right)\right)$; hence $Q_{1}^{n-1}=\sum_{k=0}^{n-1} P_{k+1} \breve{B}_{k}^{n-1}\left(z ; q^{1 / 2} a, q^{1 / 2} b ; q\right)$. Thus

$$
\begin{aligned}
\left(\mathcal{D}_{q} \breve{P}\right)(z)= & \frac{\kappa_{n, 1}(q)}{\beta_{0}-\alpha_{n-1}} \sum_{k=0}^{n-1}\left\{P_{k+1} \breve{B}_{k}^{n-1}\left(z ; q^{1 / 2} a, q^{1 / 2} b ; q\right)\right. \\
& \left.-P_{k} \breve{B}_{k}^{n-1}\left(z ; q^{-1 / 2} a, q^{-1 / 2} b ; q\right)\right\} .
\end{aligned}
$$

Setting $P_{k}=\delta_{k, j}, k=0, \ldots, n$ in (6.6) and (6.8), using that $\kappa_{n, 1}(q)=q^{-(n-1) / 2}[n]_{q}$, and applying (3.3) to simplify $\beta_{0}-\alpha_{n-1}$ yields (6.3).

Next we prove (6.4). By (6.7) and Proposition 6.2,

$$
\begin{aligned}
\left(\mathcal{D}_{q} \breve{P}\right)(z)= & \kappa_{n, 1}(q) \sum_{k=0}^{n-1} \breve{p}\left(\left(\varphi\left(q^{k-(n-2) / 2} a\right), 1\right), \ldots,\left(\varphi\left(q^{(n-2) / 2} a\right), 1\right),\right. \\
& \left.\left(\varphi\left(q^{-(n-2) / 2} b\right), 1\right), \ldots,\left(\varphi\left(q^{k-1-(n-2) / 2} b\right), 1\right), \delta\right) \breve{B}_{k}^{n-1}(z ; a, b ; q)
\end{aligned}
$$

Since the AW-blossom of a polynomial is independent of the parameters $a$ and $b$, on the right-hand side of (6.9) we can replace $a$ by $q^{1 / 2} a$ and $b$ by $q^{-1 / 2} b$. Therefore by (6.9), (6.1), (6.5), and the multilinear property of the AW-blossom,

$$
\begin{aligned}
\left(\mathcal{D}_{q} \breve{P}\right)(z)= & \kappa_{n, 1}(q) \sum_{k=0}^{n-1} \breve{p}\left(\left(\alpha_{k+1}, 1\right), \ldots,\left(\alpha_{n-1}, 1\right),\left(\beta_{0}, 1\right), \ldots,\left(\beta_{k-1}, 1\right), \delta\right) \\
& \times \breve{B}_{k}^{n-1}\left(z ; q^{1 / 2} a, q^{-1 / 2} b ; q\right) \\
= & \kappa_{n, 1}(q) \sum_{k=0}^{n-1} \frac{\left(P_{k+1}-P_{k}\right)}{\beta_{k}-\alpha_{k}} \breve{B}_{k}^{n-1}\left(z ; q^{1 / 2} a, q^{-1 / 2} b ; q\right)
\end{aligned}
$$

Equation (6.4) now follows by setting $P_{k}=\delta_{k, j}, k=0, \ldots, n$ in (6.6) and (6.10), using that $\kappa_{n, 1}(q)=q^{-(n-1) / 2}[n]_{q}$, and applying (3.3) to simplify $\beta_{j}-\alpha_{j}$. 
Next we derive two recurrences and two symmetry relations for the AW-Bernstein basis functions.

\section{Proposition 6.5 (Recurrence Relations)}

$$
\begin{aligned}
\breve{B}_{j}^{n}(z ; a, b ; q)= & \frac{\varphi\left(q^{(n-1) / 2} z\right)-\varphi\left(q^{(n-1) / 2} a\right)}{\varphi\left(q^{-(n-1) / 2} b\right)-\varphi\left(q^{(n-1) / 2} a\right)} \breve{B}_{j-1}^{n-1}\left(q^{-1 / 2} z ; q^{1 / 2} a, q^{1 / 2} b ; q\right) \\
& +\frac{\varphi\left(q^{-(n-1) / 2} b\right)-\varphi\left(q^{(n-1) / 2} z\right)}{\varphi\left(q^{-(n-1) / 2} b\right)-\varphi\left(q^{(n-1) / 2} a\right)} \breve{B}_{j}^{n-1}\left(q^{-1 / 2} z ; q^{-1 / 2} a, q^{-1 / 2} b ; q\right),
\end{aligned}
$$

$j=0, \ldots, n$, and

$$
\begin{aligned}
\breve{B}_{j}^{n}(z ; a, b ; q)= & \frac{\varphi\left(q^{-(n-1) / 2} z\right)-\varphi\left(q^{j-1-(n-1) / 2} a\right)}{\varphi\left(q^{j-1-(n-1) / 2} b\right)-\varphi\left(q^{j-1-(n-1) / 2} a\right)} \breve{B}_{j-1}^{n-1}\left(q^{1 / 2} z ; q^{1 / 2} a, q^{-1 / 2} b ; q\right) \\
& +\frac{\varphi\left(q^{j-(n-1) / 2} b\right)-\varphi\left(q^{-(n-1) / 2} z\right)}{\varphi\left(q^{j-(n-1) / 2} b\right)-\varphi\left(q^{j-(n-1) / 2} a\right)} \breve{B}_{j}^{n-1}\left(q^{1 / 2} z ; q^{1 / 2} a, q^{-1 / 2} b ; q\right),
\end{aligned}
$$

$j=0, \ldots, n$, where $\breve{B}_{-1}^{n}=0$ and $\breve{B}_{n}^{n-1}=0$.

Proof Let $\left\{\alpha_{j}\right\}_{j=0}^{n-1}$ and $\left\{\beta_{j}\right\}_{j=0}^{n-1}$ be as in (6.1), and set $\xi_{j}=\eta_{j}=1, j=0, \ldots, n-1$. First we prove (6.11). Applying the last step $(s=n)$ of the recursive evaluation algorithm from Corollary 5.2 with $\sigma=\operatorname{Id} \in S_{n}$ and $\left\{\breve{P}_{k}=\delta_{k, j}\right\}_{k=0}^{n}$ to evaluate $\breve{P}(z)=\breve{B}_{j}^{n}(z ; a, b ; q)$, we get

$$
\breve{B}_{j}^{n}(z ; a, b ; q)=\frac{\beta_{0}-\varphi\left(q^{n-1} \xi\right)}{\beta_{0}-\alpha_{n-1}} \breve{P}_{0}^{n-1}(z)+\frac{\varphi\left(q^{n-1} \xi\right)-\alpha_{n-1}}{\beta_{0}-\alpha_{n-1}} \breve{P}_{1}^{n-1}(z),
$$

where $\xi=q^{-(n-1) / 2} z$. Now notice that $\left\{q^{s-1-(n-1) / 2} z\right\}_{s=1}^{n-1}=\left\{q^{s-1-(n-2) / 2}\right.$ $\left.q^{-1 / 2} z\right\}_{s=1}^{n-1}$. Then observe that $\breve{P}_{0}^{n-1}(z)$ is generated by the recursive evaluation algorithm for degree $n-1$ polynomials with parameter sequence $\left(\alpha_{0}, \ldots, \alpha_{n-2}, \beta_{0}, \ldots\right.$, $\beta_{n-2}$, permutation $\sigma=\operatorname{Id} \in S_{n-1}$, and initial blossom values $\left\{\breve{P}_{k}^{0}=\delta_{k, j}\right\}_{k=0}^{n-1}$. Therefore $\breve{P}_{0}^{n-1}(z)=\breve{B}_{j}^{n-1}\left(q^{-1 / 2} z ; q^{-1 / 2} a, q^{-1 / 2} b ; q\right)$.

Similar observations show that $\breve{P}_{1}^{n-1}(z)$ is generated from the recursive evaluation algorithm for degree $n-1$ polynomials with parameter sequence $\left(\alpha_{1}, \ldots, \alpha_{n-1}, \beta_{1}\right.$, $\left.\ldots, \beta_{n-1}\right)$, permutation $\sigma=\operatorname{Id} \in S_{n-1}$, and initial blossom values $\left\{\breve{P}_{k}^{0}=\delta_{k, j-1}\right\}_{k=0}^{n-1}$. Therefore $\breve{P}_{1}^{n-1}(z)=\breve{B}_{j-1}^{n-1}\left(q^{-1 / 2} z ; q^{1 / 2} a, q^{1 / 2} b ; q\right)$. Substituting these expressions for $\breve{P}_{0}^{n-1}(z)$ and $\breve{P}_{1}^{n-1}(z)$ in (6.13) yields (6.11).

Now we prove (6.12). We apply the first step $(s=1)$ of the recursive evaluation algorithm from Corollary 5.2 with $\sigma=\operatorname{Id} \in S_{n}$ and $\left\{\breve{P}_{k}^{0}=\delta_{k, j}\right\}_{k=0}^{n}$ to evaluate $\breve{P}(z)=\breve{B}_{j}^{n}(z ; a, b ; q)$. By (5.5), $\breve{P}_{k}^{1}(z)=0, k \neq j-1, j$. Therefore, again by (5.5),

$$
\breve{B}_{j}^{n}(z ; a, b ; q)=\breve{P}_{j-1}^{1}(z) \breve{R}(z)+\breve{P}_{j}^{1}(z) \breve{S}(z)
$$


where $\breve{R}(z)$ and $\breve{S}(z)$ are generated by the components of this recursive evaluation algorithm that originate from $\breve{P}_{j-1}^{1}(z)$ and $\breve{P}_{j}^{1}(z)$, and

$$
\breve{P}_{j-1}^{1}(z)=\frac{\varphi(\xi)-\alpha_{j-1}}{\beta_{j-1}-\alpha_{j-1}}, \quad \breve{P}_{j}^{1}(z)=\frac{\beta_{j}-\varphi(\xi)}{\beta_{j}-\alpha_{j}}
$$

Notice also that $\left\{q^{s-1-(n-1) / 2} z\right\}_{s=2}^{n}=\left\{q^{s-1-(n-2) / 2} q^{1 / 2} z\right\}_{s=1}^{n-1}$. Then $\breve{R}(z)$ and $\breve{S}(z)$ are generated by the recursive evaluation algorithms of Corollary 5.2 with parameter sequence

$$
\begin{aligned}
& \left(\alpha_{1}, \ldots, \alpha_{n-1}, \beta_{0}, \ldots, \beta_{n-2}\right) \\
& \quad=\left(\varphi\left(q^{-(n-2) / 2} q^{1 / 2} a\right), \ldots, \varphi\left(q^{(n-2) / 2} q^{1 / 2} a\right), \varphi\left(q^{-(n-2) / 2} q^{-1 / 2} b\right), \ldots, \varphi\left(q^{(n-2) / 2} q^{-1 / 2} b\right)\right)
\end{aligned}
$$

and with $\sigma=\operatorname{Id} \in S_{n-1}$, initial blossom values $\left\{\breve{R}_{k}^{0}=\delta_{k, j-1}\right\}_{k=0}^{n-1}$ and $\left\{\breve{S}_{k}^{0}=\delta_{k, j}\right\}_{k=0}^{n-1}$, and $z$ replaced by $q^{1 / 2} z$. Thus

$$
\breve{R}(z)=\breve{B}_{j-1}^{n-1}\left(q^{1 / 2} z ; q^{1 / 2} a, q^{-1 / 2} b ; q\right), \quad \breve{S}(z)=\breve{B}_{j}^{n-1}\left(q^{1 / 2} z ; q^{1 / 2} a, q^{-1 / 2} b ; q\right)
$$

Relation (6.12) follows from (6.14), (6.15), and (6.16).

Proposition 6.6 (Symmetry Relations)

$$
\begin{aligned}
\breve{B}_{k}^{n}(z ; a, b ; q) & =\breve{B}_{k}^{n}(z ; 1 / a, 1 / b ; 1 / q), \quad k=0, \ldots, n, \\
\breve{B}_{k}^{n}(z ; a, b ; q) & =\breve{B}_{n-k}^{n}(z ; b, a ; 1 / q), \quad k=0, \ldots, n .
\end{aligned}
$$

Proof Relation (6.17) follows from the dual functional property (6.2) and the observation that

$$
\begin{aligned}
\breve{p}( & \left.\varphi\left(q^{k-(n-1) / 2} a\right), \ldots, \varphi\left(q^{(n-1) / 2} a\right), \varphi\left(q^{-(n-1) / 2} b\right), \ldots, \varphi\left(q^{k-1-(n-1) / 2} b\right)\right) \\
= & \breve{p}\left(\varphi\left((1 / q)^{k-(n-1) / 2} / a\right), \ldots, \varphi\left((1 / q)^{(n-1) / 2} / a\right), \varphi\left((1 / q)^{-(n-1) / 2} / b\right), \ldots,\right. \\
& \left.\varphi\left((1 / q)^{k-1-(n-1) / 2} / b\right)\right),
\end{aligned}
$$

$k=0, \ldots, n$, which is due to the fact that $\varphi(w)=\varphi(1 / w)$.

Relation (6.18) follows from the dual functional property (6.2) and the observation that

$$
\begin{aligned}
& \breve{p}\left(\varphi\left(q^{k-(n-1) / 2} a\right), \ldots, \varphi\left(q^{(n-1) / 2} a\right), \varphi\left(q^{-(n-1) / 2} b\right), \ldots, \varphi\left(q^{k-1-(n-1) / 2} b\right)\right) \\
& \quad=\breve{p}\left(\varphi\left((1 / q)^{n-k-(n-1) / 2} b\right), \ldots, \varphi\left((1 / q)^{(n-1) / 2} b\right), \varphi\left((1 / q)^{-(n-1) / 2} a\right), \ldots, \varphi\left((1 / q)^{n-k-1-(n-1) / 2} a\right)\right),
\end{aligned}
$$

$k=0, \ldots, n$, which is due to the symmetry of the AW-blossom.

Using recurrence relation (6.11) (or (6.12) but the derivation is harder) and induction on $n$, next we derive an explicit formula for the Askey-Wilson Bernstein basis functions. 
Proposition 6.7 (Explicit Formula)

$$
\breve{B}_{k}^{n}(z ; a, b ; q)=\frac{(-1)^{k}}{(a b)^{k}} q^{-\left(\begin{array}{c}
k \\
2
\end{array}\right)}\left[\begin{array}{l}
n \\
k
\end{array}\right]_{q} \frac{(a z, a / z ; q)_{k}\left(b^{-1} z, b^{-1} / z ; q\right)_{n-k}}{(a / b ; q)_{n}\left(q^{n-k} /(a b) ; 1 / q\right)_{k}\left(q^{-k} /(a b) ; q\right)_{n-k}},
$$

$k=0, \ldots, n$.

Proof Set $\breve{B}_{-1}^{n}=\breve{B}_{n+1}^{n}=0$. We will use induction on $n$ and recurrence relation (6.11). By definition $\breve{B}_{0}^{0}=1$, and also by the definition of $\left\{\breve{B}_{k}^{1}\right\}_{k=0}^{1},(6.11)$, and (3.4), it follows that

$$
\begin{aligned}
& \breve{B}_{0}^{1}(z)=\frac{\varphi(b)-\varphi(z)}{\varphi(b)-\varphi(a)}=\frac{(1-z / b)(1-1 /(b z))}{(1-a / b)(1-1 /(a b))}, \\
& \breve{B}_{1}^{1}(z)=\frac{\varphi(z)-\varphi(a)}{\varphi(b)-\varphi(a)}=\frac{(1-a z)(1-a / z)}{(1-a b)(1-a / b)},
\end{aligned}
$$

which verify (6.19) when $n=1$.

Now assume that (6.19) holds for some $n \in \mathbb{N}$ and all $k=0, \ldots, n$. By (6.11), (3.3), and (6.19),

$$
\begin{aligned}
& \breve{B}_{k}^{n+1}(z ; a, b ; q) \\
& =\frac{(z-a)\left(1 / z-q^{n} a\right)}{\left(b-q^{n} a\right)(1 / b-a)} \breve{B}_{k-1}^{n}\left(q^{-1 / 2} z ; q^{1 / 2} a, q^{1 / 2} b ; q\right) \\
& +\frac{\left(q^{n} z-b\right)(1 / z-b)}{\left(q^{n} a-b\right)(1 / a-b)} \breve{B}_{k}^{n}\left(q^{-1 / 2} z ; q^{-1 / 2} a, q^{-1 / 2} b ; q\right) \\
& =\frac{(-1)^{k-1} q^{-\left(\begin{array}{c}
k-1 \\
2
\end{array}\right)}}{(q a b)^{k-1}}\left[\begin{array}{c}
n \\
k-1
\end{array}\right]_{q} \frac{(1-a / z)\left(1-q^{n} a z\right)}{\left(1-q^{n} a / b\right)(1-a b)} \\
& \times \frac{(a z, q a / z ; q)_{k-1}\left(q^{-1} b^{-1} z, b^{-1} / z ; q\right)_{n+1-k}}{(a / b ; q)_{n}\left(q^{n-k} /(a b) ; 1 / q\right)_{k-1}\left(q^{-k} /(a b) ; q\right)_{n+1-k}} \\
& +\frac{(-1)^{k} q^{-\left(\begin{array}{c}
k \\
2
\end{array}\right)}}{\left(q^{-1} a b\right)^{k}}\left[\begin{array}{l}
n \\
k
\end{array}\right]_{q} \frac{\left(1-q^{n} b^{-1} z\right)\left(1-b^{-1} / z\right)}{\left(1-q^{n} a / b\right)(1-1 /(a b))} \\
& \times \frac{\left(q^{-1} a z, a / z ; q\right)_{k}\left(b^{-1} z, q b^{-1} / z ; q\right)_{n-k}}{(a / b ; q)_{n}\left(q^{n+1-k} /(a b) ; 1 / q\right)_{k}\left(q^{1-k} /(a b) ; q\right)_{n-k}} \\
& =\frac{(-1)^{k} q^{-\left(\begin{array}{c}
k \\
2
\end{array}\right)}}{(a b)^{k}(1-1 /(a b))} \frac{(q ; q)_{n}}{(q ; q)_{k}(q ; q)_{n+1-k}} \\
& \times \frac{(a z ; q)_{k-1}(a / z ; q)_{k}\left(b^{-1} z ; q\right)_{n-k}\left(b^{-1} / z ; q\right)_{n+1-k}}{(a / b ; q)_{n+1}\left(q^{n+1-k} /(a b) ; 1 / q\right)_{k}\left(q^{-k} /(a b) ; q\right)_{n+1-k}} \\
& \times\left\{\left(1-q^{k}\right)\left(1-q^{n} a z\right)\left(1-q^{-1} b^{-1} z\right)\left(1-q^{n+1-k} /(a b)\right)\right. \\
& \left.+q^{k}\left(1-q^{n+1-k}\right)\left(1-q^{-1} a z\right)\left(1-q^{n} b^{-1} z\right)\left(1-q^{-k} /(a b)\right)\right\} \text {. }
\end{aligned}
$$


Let $Q(z)$ denote the quadratic polynomial in the last two lines of (6.20). Notice that

$$
\begin{aligned}
Q\left(q^{1-k} / a\right)= & \left(1-q^{n+1-k}\right)\left(1-q^{-k} /(a b)\right)\left(1-q^{n+1-k} /(a b)\right) \\
& \times\left[\left(1-q^{k}\right)+q^{k}\left(1-q^{-k}\right)\right]=0
\end{aligned}
$$

and

$$
\begin{aligned}
Q\left(q^{k-n} b\right)= & \left(1-q^{k}\right)\left(1-q^{k} a b\right)\left(1-q^{k-n-1}\right)\left(1-q^{n+1-k} /(a b)\right) \\
& \times\left[1+q^{k}\left(-q^{n+1-k}\right)\left(-q^{k-n-1} a b\right)\left(-q^{-k} /(a b)\right)\right]=0 .
\end{aligned}
$$

Thus $Q(z)=\gamma\left(1-q^{k-1} a z\right)\left(1-q^{n-k} b^{-1} z\right)$, where

$$
\begin{aligned}
q^{n-1} a b^{-1} \gamma= & \left(1-q^{k}\right)\left(1-q^{n+1-k} /(a b)\right) q^{n-1} a b^{-1} \\
& +\left(1-q^{n+1-k}\right)\left(q^{k}-1 /(a b)\right) q^{n-1} a b^{-1} .
\end{aligned}
$$

Solving for $\gamma$ yields $\gamma=1+q^{n+1} /(a b)-1 /(a b)-q^{n+1}=\left(1-q^{n+1}\right)(1-1 /(a b))$. Therefore,

$$
Q(z)=\left(1-q^{n+1}\right)(1-1 /(a b))\left(1-q^{k-1} a z\right)\left(1-q^{n-k} b^{-1} z\right)
$$

Replacing the expression in the last two lines of (6.20) by (6.21) yields (6.19) for $\breve{B}_{k}^{n+1}(z ; a, b ; q)$.

We end this section with a degree elevation formula for the AW-Bernstein basis functions.

\section{Corollary 6.8 (Degree Elevation Formula)}

$$
\breve{B}_{k}^{n}(z ; a, b ; q)=c_{n, k} \breve{B}_{k}^{n+1}(z ; a, b ; q)+d_{n, k} \breve{B}_{k+1}^{n+1}(z ; a, b ; q)
$$

where

$$
\begin{aligned}
c_{n, k}= & \frac{\left(1-q^{n-k+1}\right)\left(1-q^{n-k+1} /(a b)\right)}{\left(1-q^{n+1}\right)\left(1-q^{n-2 k+1} /(a b)\right)}, \\
d_{n, k}= & q^{n-k} \frac{\left(1-q^{k+1}\right)\left(1-q^{-k-1} /(a b)\right)}{\left(1-q^{n+1}\right)\left(1-q^{n-2 k-1} /(a b)\right)} .
\end{aligned}
$$

Proof To simplify our notation, we set $\lambda=1 /(a b)$. Substituting formula (6.19) for the AW-Bernstein basis functions in (6.22) and dividing out by all nonvanishing common factors, we obtain

$$
1=\tilde{c}_{n, k}\left(1-q^{n-k} b^{-1} z\right)\left(1-q^{n-k} b^{-1} / z\right)+\tilde{d}_{n, k}\left(1-q^{k} a z\right)\left(1-q^{k} a / z\right),
$$


Equating the constant coefficients and the coefficients of $z+1 / z$ on both sides of (6.24) yields the system

$$
\left\{\begin{array}{c}
\left(1+\beta^{2}\right) \tilde{c}_{n, k}+\left(1+\alpha^{2}\right) \tilde{d}_{n, k}=1 \\
\beta \tilde{c}_{n, k}+\alpha \tilde{d}_{n, k}=0
\end{array}\right.
$$

with $\alpha=q^{k} a, \beta=q^{n-k} b^{-1}$, whose solution is

$$
\begin{aligned}
& \tilde{c}_{n, k}=\alpha /[(\alpha-\beta)(1-\alpha \beta)]=1 /\left[\left(1-q^{n} a / b\right)\left(1-q^{n-2 k} \lambda\right)\right] \\
& \tilde{d}_{n, k}=-\beta /[(\alpha-\beta)(1-\alpha \beta)]=-q^{n-2 k} \lambda /\left[\left(1-q^{n} a / b\right)\left(1-q^{n-2 k} \lambda\right)\right] .
\end{aligned}
$$

Equations (6.23) follow from (6.25) and (6.26).

\section{Identities}

In this section we derive identities for the Askey-Wilson Bernstein basis functions using the Askey-Wilson blossom and its dual functional property. These identities are the Askey-Wilson analogues of standard identities for the classical Bernstein basis functions. We will then use these identities to provide simple derivations of two nontrivial basic hypergeometric sums.

We begin with an alternative representation for $\breve{B}_{k}^{n}(z ; a, b ; q)$, which is more convenient for proving identities for basic hypergeometric series.

\section{Proposition 7.1}

$$
\breve{B}_{k}^{n}(z ; a, b ; q)=\frac{\left(b^{-1} z, b^{-1} / z ; q\right)_{n}}{(1 /(a b), a / b ; q)_{n}} \frac{\left(q^{-n}, q^{-n} a b, q^{1-\frac{n}{2}} \sqrt{a b},-q^{1-\frac{n}{2}} \sqrt{a b}, a z, a / z ; q\right)_{k}}{\left(q, q a b, q^{-\frac{n}{2}} \sqrt{a b},-q^{-\frac{n}{2}} \sqrt{a b}, q^{-n+1} b z, q^{-n+1} b / z ; q\right)_{k}}(q b / a)^{k},
$$

$k=0, \ldots, n$.

Proof We will use the identity

$$
(A ; q)_{n-k}=(A ; q)_{n}(-1 / A)^{k} q^{\left(\begin{array}{c}
n-k \\
2
\end{array}\right)-\left(\begin{array}{c}
n \\
2
\end{array}\right)} /\left(q^{-n+1} / A ; q\right)_{k}, \quad k=0, \ldots, n .
$$

\section{Springer}


421

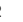

-

Then

$$
\begin{aligned}
\left(q^{n-k} \lambda ; 1 / q\right)_{k}\left(q^{-k} \lambda ; q\right)_{n-k}= & \frac{\left(q^{-k} \lambda ; q\right)_{n+1}}{\left(1-q^{n-2 k} \lambda\right)}=\frac{\left(q^{-k} \lambda ; q\right)_{k}(1-\lambda)(q \lambda ; q)_{n-k}}{\left(1-q^{n-2 k} \lambda\right)} \\
= & (-\lambda / q)^{k} q^{-\left(\begin{array}{c}
k \\
2
\end{array}\right)}(q / \lambda ; q)_{k}(q \lambda ; q)_{n}(-1 /(q \lambda))^{k} \\
& \times \frac{q^{\left(\begin{array}{c}
n-k \\
2
\end{array}\right)-\left(\begin{array}{c}
n \\
2
\end{array}\right)}}{\left(q^{-n} / \lambda ; q\right)_{k}} \frac{(1-\lambda)}{\left(1-q^{n-2 k} \lambda\right)},
\end{aligned}
$$

where in the last step we applied (7.2) with $A=q \lambda$. Next we use the identity

$$
\left(1-q^{2 k} B\right)=\left(1-q^{k} \sqrt{B}\right)\left(1+q^{k} \sqrt{B}\right)=(1-B) \frac{(q \sqrt{B},-q \sqrt{B} ; q)_{k}}{(\sqrt{B},-\sqrt{B} ; q)_{k}},
$$

with $B=q^{-n} / \lambda$ to write

$$
\begin{aligned}
\left(1-q^{n-2 k} \lambda\right)= & -q^{n-2 k} \lambda\left(1-q^{2 k-n} / \lambda\right)=q^{-2 k}\left(1-q^{n} \lambda\right) \\
& \times \frac{\left(q^{1-n / 2} / \sqrt{\lambda},-q^{1-n / 2} / \sqrt{\lambda} ; q\right)_{k}}{\left(q^{-n / 2} / \sqrt{\lambda},-q^{-n / 2} / \sqrt{\lambda} ; q\right)_{k}} .
\end{aligned}
$$

Combining (7.3) and (7.4), we get

$$
\begin{aligned}
\left(q^{n-k} \lambda ; 1 / q\right)_{k}\left(q^{-k} \lambda ; q\right)_{n-k}= & q^{-\left(\begin{array}{c}
k \\
2
\end{array}\right)+\left(\begin{array}{c}
n-k \\
2
\end{array}\right)-\left(\begin{array}{c}
n \\
2
\end{array}\right)}(\lambda ; q)_{n} \\
& \times \frac{\left(q / \lambda, q^{-n / 2} / \sqrt{\lambda},-q^{-n / 2} / \sqrt{\lambda} ; q\right)_{k}}{\left(q^{-n} / \lambda, q^{1-n / 2} / \sqrt{\lambda},-q^{1-n / 2} / \sqrt{\lambda} ; q\right)_{k}} .
\end{aligned}
$$

Also by (7.2),

$$
\begin{aligned}
\frac{\left(b^{-1} z, b^{-1} / z ; q\right)_{n-k}}{(q ; q)_{n-k}}= & \frac{\left(b^{-1} z, b^{-1} / z ; q\right)_{n}}{(q ; q)_{n}}\left(-q b^{2}\right)^{k} q^{\left(\begin{array}{c}
n-k \\
2
\end{array}\right)-\left(\begin{array}{c}
n \\
2
\end{array}\right)} \\
& \times \frac{\left(q^{-n} ; q\right)_{k}}{\left(q^{-n+1} b / z, q^{-n+1} b z ; q\right)_{k}}
\end{aligned}
$$

Substituting (7.5) with $\lambda=1 /(a b)$ and (7.6) into (6.19) and simplifying yields (7.1).

\section{Proposition 7.2 (Partition of Unity)}

$$
\sum_{j=0}^{n} \breve{B}_{j}^{n}(z ; a, b ; q)=1
$$

Proof Equation (7.7) follows from the dual functional property (6.2) applied to the constant polynomial $P(x)=1$ whose AW-blossom is $\breve{p}\left(u_{1}, \ldots, u_{n}\right)=1$. 
An immediate consequence of Proposition 7.2 is the following summation formula for a ${ }_{6} \phi_{5}$ series.

\section{Corollary 7.3}

$$
\begin{aligned}
& { }_{6} \phi_{5}\left(\begin{array}{l}
q^{-n}, q^{-n} a b, q^{1-n / 2} \sqrt{a b},-q^{1-n / 2} \sqrt{a b}, a z, a / z \\
q a b, q^{-n / 2} \sqrt{a b},-q^{-n / 2} \sqrt{a b}, q^{1-n} b z, q^{1-n} b / z
\end{array} \mid q, q b / a\right) \\
& =\frac{(1 /(a b), a / b ; q)_{n}}{\left(b^{-1} z, b^{-1} / z ; q\right)_{n}} .
\end{aligned}
$$

Proof Substituting (7.1) into (7.7) yields (7.8).

Formula (7.8) is equivalent to the terminating version of Rogers' ${ }_{6} \phi_{5}$ sum [10, $(12.2 .31)]$

$$
{ }_{6} \phi_{5}\left(\begin{array}{c}
A, q \sqrt{A},-q \sqrt{A}, B, C, q^{-n} \\
\sqrt{A},-\sqrt{A}, q A / B, q A / C, q^{n+1} A
\end{array} \mid q, q^{n+1} \frac{A}{B C}\right)=\frac{(q A, q A /(B C) ; q)_{n}}{(q A / B, q A / C ; q)_{n}} .
$$

To verify this equivalence, set in (7.9) $A=q^{-n} a b, B=a z$, and $C=a / z$. With this substitution the left-hand side of (7.9) becomes the left-hand side of (7.8). The right-hand side of (7.9) becomes $\frac{\left(q^{1-n} a b, q^{1-n} b / a ; q\right)_{n}}{\left(q^{1-n} b / z, q^{1-n} b z ; q\right)_{n}}$, which reduces to the right-hand side of (7.8) after applying the identity

$$
\left(q^{1-n} F ; q\right)_{n}=q^{-\left(\begin{array}{l}
n \\
2
\end{array}\right)}(-F)^{n}(1 / F ; q)_{n}
$$

with $F=a b, F=b / a, F=b / z$, and $F=b z$.

M. Ismail introduced a simple and clever argument to extend some terminating basic hypergeometric sums to their nonterminating analogs, and in particular to extend (7.9) to its nonterminating analog $[10,(12.2 .28)]$. M. Ismail observed that the difference between the left- and right-hand sides of $[10,(12.2 .28)]$ is an analytic function of $1 / d$ in an open set containing 0 that vanishes at $d=q^{-n}, n \in \mathbb{N}$, since [10, (12.2.28)] reduces to $[10,(12.2 .31)]$ when $d=q^{-n}, n \in \mathbb{N}$. Hence, by the uniqueness theorem for analytic functions $[19, \S 5.8$, Theorem 22$]$ this function vanishes, that is, $[10,(12.2 .28)]$ holds in an open set containing 0 .

A fundamental identity associated with any polynomial blossom is the Marsden identity. The Marsden identity is usually derived by applying the dual functional property of the blossom to the polynomial whose roots are the functions that appear in the diagonal property of the blossom.

\section{Proposition 7.4 (Marsden Identity)}

$$
\begin{aligned}
& \left(q^{-(n-1) / 2} z / w, q^{-(n-1) / 2} /(z w) ; q\right)_{n}(-w)^{n} \\
& =\sum_{k=0}^{n}\left(q^{-(n-1) / 2} b w, q^{-(n-1) / 2} b / w ; q\right)_{k} \\
& \quad \times\left(q^{k-(n-1) / 2} a w, q^{k-(n-1) / 2} a / w ; q\right)_{n-k} a^{-n+k} b^{-k} \breve{B}_{k}^{n}(z ; a, b ; q) .
\end{aligned}
$$


Proof For a fixed $y$ consider the polynomial $\prod_{j=0}^{n-1}\left(y-\varphi\left(q^{j-(n-1) / 2} z\right)\right)$ in $x=\varphi(z)$, whose AW-blossom is $\prod_{j=1}^{n}\left(y-u_{j}\right)$. By the dual functional property (6.2),

$$
\begin{aligned}
& \prod_{j=0}^{n-1}\left(y-\varphi\left(q^{j-(n-1) / 2} z\right)\right)=\sum_{k=0}^{n} \prod_{l=k}^{n-1}\left(y-\varphi\left(q^{l-(n-1) / 2} a\right)\right) \\
& \quad \times \prod_{l=0}^{k-1}\left(y-\varphi\left(q^{l-(n-1) / 2} b\right)\right) \breve{B}_{k}^{n}(z ; a, b ; q) .
\end{aligned}
$$

Set $y=\varphi(w)$ in (7.12). By (3.4), the left-hand side of (7.12) becomes

$$
\begin{aligned}
& \prod_{j=0}^{n-1}\left(\varphi(w)-\varphi\left(q^{j-(n-1) / 2} z\right)\right)=(w / 2)^{n} \prod_{j=0}^{n-1}\left[\left(1-q^{j-(n-1) / 2} z / w\right)\right. \\
& \left.\quad \times\left(1-q^{-j+(n-1) / 2} /(z w)\right)\right] \\
& =(w / 2)^{n}\left(q^{-(n-1) / 2} z / w, q^{-(n-1) / 2} /(z w) ; q\right)_{n} .
\end{aligned}
$$

Similarly, with $y=\varphi(w)$, by (3.4) the coefficient of $\breve{B}_{k}^{n}(z ; a, b ; q)$ in (7.12) becomes

$$
\begin{aligned}
(-1 /(2 a))^{n-k} q^{(n-k)(n-1) / 2-\left(\begin{array}{c}
n \\
2
\end{array}\right)+\left(\begin{array}{c}
k \\
2
\end{array}\right)} \prod_{l=k}^{n-1}\left[\left(1-q^{l-(n-1) / 2} a w\right)\left(1-q^{l-(n-1) / 2} a / w\right)\right] \\
\quad \times(-1 /(2 b))^{k} q^{k(n-1) / 2-\left(\begin{array}{c}
k \\
2
\end{array}\right)} \prod_{l=0}^{k-1}\left[\left(1-q^{l-(n-1) / 2} b w\right)\left(1-q^{l-(n-1) / 2} b / w\right)\right] \\
=(-1 / 2)^{n} a^{-n+k} b^{-k}\left(q^{k-(n-1) / 2} a w, q^{k-(n-1) / 2} a / w ; q\right)_{n-k} \\
\quad \times\left(q^{-(n-1) / 2} b w, q^{-(n-1) / 2} b / w ; q\right)_{k} .
\end{aligned}
$$

Now (7.11) follows from (7.12), (7.13), and this form of the coefficient of $\breve{B}_{k}^{n}(z ; a, b ; q)$ in (7.12).

\section{Corollary 7.5}

${ }_{8} \phi_{7}\left(\begin{array}{c}q^{-n}, q^{-n} a b, q^{1-\frac{n}{2}} \sqrt{a b},-q^{1-\frac{n}{2}} \sqrt{a b}, a z, a / z, q^{-(n-1) / 2} b w, q^{-(n-1) / 2} b / w \\ q a b, q^{-\frac{n}{2}} \sqrt{a b},-q^{-\frac{n}{2}} \sqrt{a b}, q^{-n+1} b z, q^{-n+1} b / z, q^{-(n-1) / 2} a w, q^{-(n-1) / 2} a / w\end{array} \mid q, q\right)$

$$
=\frac{\left(1 /(a b), a / b, q^{-(n-1) / 2} z / w, q^{-(n-1) / 2} /(z w) ; q\right)_{n}}{\left(b^{-1} z, b^{-1} / z, q^{-(n-1) / 2} a w, q^{-(n-1) / 2} a / w ; q\right)_{n}}(-a w)^{n} .
$$

Proof We apply the identity $\left(q^{k} A ; q\right)_{n-k}=(A ; q)_{n} /(A ; q)_{k}$ on the right-hand side of (7.11) to write

$$
\left(q^{k-(n-1) / 2} a w, q^{k-(n-1) / 2} a / w ; q\right)_{n-k}=\frac{\left(q^{-(n-1) / 2} a w, q^{-(n-1) / 2} a / w ; q\right)_{n}}{\left(q^{-(n-1) / 2} a w, q^{-(n-1) / 2} a / w ; q\right)_{k}},
$$


and then we substitute $\breve{B}_{k}^{n}(z ; a, b ; q)$ by the expression on the right-hand side of (7.1). The resulting sum on the right-hand side of (7.11) is the ${ }_{8} \phi_{7}$ sum on the left-hand side of (7.14) times $\frac{\left(b^{-1} z, b^{-1} / z, q^{-(n-1) / 2} a w, q^{-(n-1) / 2} a / w ; q\right)_{n}}{(1 /(a b), a / b ; q)_{n}} a^{-n}$. Dividing both sides of the resulting equation by this expression yields (7.14).

The basic hypergeometric form (7.14) of the Marsden identity (7.11) is a summation formula for a very-well-poised terminating ${ }_{8} W_{7}$ series, since the ${ }_{8} \phi_{7}$ sum in (7.14) is

$$
{ }_{8} W_{7}\left(q^{-n} a b ; q^{-n}, a z, a / z, q^{-(n-1) / 2} b w, q^{-(n-1) / 2} b / w ; q\right) .
$$

More specifically, (7.14) is equivalent to Jackson's $q$-analogue of Dougall's ${ }_{7} F_{6}$ sum [3, (II.22)]

$$
\begin{aligned}
{ }_{8} \phi_{7}\left(\begin{array}{c}
A, q \sqrt{A},-q \sqrt{A}, B, C, D, E, q^{-n} \\
\sqrt{A},-\sqrt{A}, q A / B, q A / C, q A / D, q A / E, q^{n+1} A
\end{array} \mid q, q\right) \\
=\frac{(q A, q A /(B C), q A /(B D), q A /(C D) ; q)_{n}}{(q A / B, q A / C, q A / D, q A /(B C D) ; q)_{n}}
\end{aligned}
$$

where $q A^{2}=q^{-n} B C D E$. Indeed, set $A=q^{-n} a b, B=a z, C=a / z$, $D=q^{-(n-1) / 2} b w$, and $E=q^{-(n-1) / 2} b / w$ in (7.15). The left-hand side of (7.15) becomes the left-hand side of (7.14), and the right-hand side of (7.15) becomes $\frac{\left(q^{1-n} a b, q^{1-n} b / a, q^{-(n-1) / 2} /(z w), q^{-(n-1) / 2} z / w ; q\right)_{n}}{\left(q^{1-n} b / z, q^{1-n} b z, q^{-(n-1) / 2} a / w, q^{-(n-1) / 2} /(a w) ; q\right)_{n}}$. This expression reduces to the right-hand side of (7.14) after applying identity (7.10) with $F=a b, F=b / a, F=b / z, F=b z$, and $F=q^{(n-1) / 2} /(a w)$.

Proposition 7.6 (Change of Basis Formula) Let $\left\{F_{n, k}(x ; q)\right\}$ be the polynomials defined by (4.3), and let $\left\{\alpha_{l}\right\}$ and $\left\{\beta_{l}\right\}$ be the numbers defined by (6.1). Then

$$
\breve{F}_{n, k}(z ; q)=\sum_{j=0}^{n} e_{n, k}\left(\alpha_{j}, \ldots, \alpha_{n-1}, \beta_{0}, \ldots, \beta_{j-1}\right) \breve{B}_{j}^{n}(z ; a, b ; q), \quad k=0, \ldots, n .
$$

Proof By (4.3) the AW-blossom of $F_{n, k}(x ; q)=\breve{F}_{n, k}(z ; q)$ is the elementary symmetric function $e_{n, k}\left(u_{1}, \ldots, u_{n}\right)$. Then (7.16) follows from the dual functional property (6.2).

The case $k=0$ of Proposition 7.6 is the partition of unity property (7.7). Here we consider the case $k=1$ of Proposition 7.6 to derive a nontrivial identity for hypergeometric series. By (7.1),

$$
\begin{aligned}
G_{n}(z, t ; a, b ; q):= & \sum_{k=0}^{n} \breve{B}_{k}^{n}(z ; a, b ; q) t^{k}=\frac{\left(b^{-1} z, b^{-1} / z ; q\right)_{n}}{(1 /(a b), a / b ; q)_{n}} \\
& \times{ }_{6} \phi_{5}\left(\begin{array}{l}
q^{-n}, q^{-n} a b, q^{1-n / 2} \sqrt{a b},-q^{1-n / 2} \sqrt{a b}, a z, a / z \\
q a b, q^{-n / 2} \sqrt{a b},-q^{-n / 2} \sqrt{a b}, q^{1-n} b z, q^{1-n} b / z
\end{array} \mid q, \frac{q b}{a} t\right) .
\end{aligned}
$$




\section{Corollary 7.7}

$$
\begin{aligned}
& \frac{(a-b)}{\left(1-q^{n}\right)} G_{n}(z, q ; a, b ; q)+\frac{(1 / a-1 / b)}{\left(1-q^{-n}\right)} G_{n}(z, 1 / q ; a, b ; q) \\
& =z+\frac{1}{z}-a-\frac{1}{a}-\frac{b-a}{1-q^{n}}-\frac{1 / b-1 / a}{1-q^{-n}} .
\end{aligned}
$$

Proof A straightforward computation shows that

$$
\begin{aligned}
e_{n, 1}\left(\alpha_{j}, \ldots, \alpha_{n-1}, \beta_{0}, \ldots, \beta_{j-1}\right)= & q^{-(n-1) / 2} \frac{[n]_{q}}{2} \\
& \times\left\{a+\frac{1}{a}+\frac{[j]_{q}}{[n]_{q}}(b-a)+\frac{[j]_{1 / q}}{[n]_{1 / q}}\left(\frac{1}{b}-\frac{1}{a}\right)\right\},
\end{aligned}
$$

$j=0, \ldots, n$. Substituting (4.6) and (7.19) into the case $k=1$ of (7.16) and then applying (7.7) and (7.17) to simplify the resulting expression yields (7.18).

Degree elevation is an important procedure in the theory of Bézier curves and surfaces. The convergence and rate of convergence of the degree elevated control polygons of a Bézier curve, and finding the limit curve, are challenging problems in approximation theory. These degree elevated control polygons converge uniformly to the Bézier curve in the classical case [4], and to a certain polygon in the quantum $(q, h)$ case [7]. But the convergence and rate of convergence of the degree elevated control polygons are open questions for AW-Bézier curves. Here we give a general formula for the control points of a degree elevated AW-Bézier curve, which may serve as a starting point for studying the convergence of the degree elevated control polygons of AW-Bézier curves.

Proposition 7.8 Let $\breve{P}(z)$ be a degree $n$ AW-Bézier curve with control points $\left\{P_{k}^{0}\right\}_{k=0}^{n}$. Then for every $m \in \mathbb{N}, \breve{P}(z)$ is a degree $n+m$ AW-Bézier curve with control points $\left\{P_{k}^{m}\right\}_{k=0}^{n+m}$ given by

$$
P_{k}^{m}=\sum_{s=0}^{m} f_{n, k, k-s}^{m} P_{k-s}^{0}, \quad k=0, \ldots, n+m,
$$

where $P_{j}^{0}=0$ for $j \notin\{0, \ldots, n\}, f_{n, k, j}^{m}=0$ for $j \notin\{0, \ldots, n\}$, and

$$
\begin{aligned}
f_{n, k, k-s}^{m}= & q^{s(n-k+s)}\left[\begin{array}{c}
m \\
s
\end{array}\right]_{q} \\
& \times \frac{\left(q^{k-s+1}, q^{-k} /(a b) ; q\right)_{s}\left(q^{n+s-k+1}, q^{n+s-k+1} /(a b) ; q\right)_{m-s}}{\left(q^{n+1} ; q\right)_{m}\left(q^{n-2 k+s} /(a b) ; q\right)_{s}\left(q^{n-2 k+2 s+1} /(a b) ; q\right)_{m-s}},
\end{aligned}
$$


$s=0, \ldots, \min \{k, m\}$. Moreover,

$$
\sum_{s=0}^{m} f_{n, k, k-s}^{m}=1, \quad k=0, \ldots, n+m
$$

Proof We will use induction on $m \in \mathbb{N}$. By Corollary 6.8,

$$
\begin{aligned}
\breve{P}(z) & =\sum_{k=0}^{n} P_{k}^{0}\left(c_{n, k} \breve{B}_{k}^{n+1}(z ; a, b ; q)+d_{n, k} \breve{B}_{k+1}^{n+1}(z ; a, b ; q)\right) \\
& =\sum_{k=0}^{n+1} P_{k}^{1} \breve{B}_{k}^{n+1}(z ; a, b ; q)
\end{aligned}
$$

where $P_{k}^{1}=c_{n, k} P_{k}^{0}+d_{n, k-1} P_{k-1}^{0}, k=0, \ldots, n+1$. Thus (7.20) holds in this case with

$$
\begin{aligned}
& f_{n, k, k}^{1}=c_{n, k}, \quad f_{n, k, k-1}^{1}=d_{n, k-1}, \quad k=1, \ldots, n, \\
& f_{n, 0,0}^{1}=c_{n, 0}=1, \quad f_{n, n+1, n}^{1}=d_{n, n}=1 .
\end{aligned}
$$

Then (7.23) and (6.23) show that (7.21) holds for $m=1$, and by (6.23) we have

$$
\begin{aligned}
& f_{n, k, k}^{1}+f_{n, k, k-1}^{1}=c_{n, k}+d_{n, k-1} \\
& \quad=\frac{\left(1-q^{n-k+1}\right)\left(1-q^{n-k+1} \lambda\right)+q^{n-k+1}\left(1-q^{k}\right)\left(1-q^{-k} \lambda\right)}{\left(1-q^{n+1}\right)\left(1-q^{n-2 k+1} \lambda\right)}=1,
\end{aligned}
$$

$$
\begin{aligned}
\breve{P}(z) & =\sum_{k=0}^{n+m} P_{k}^{m}\left(c_{n+m, k} \breve{B}_{k}^{n+m+1}(z ; a, b ; q)+d_{n+m, k} \breve{B}_{k+1}^{n+m+1}(z ; a, b ; q)\right) \\
& =\sum_{k=0}^{n+m+1} P_{k}^{m+1} \breve{B}_{k}^{n+m+1}(z ; a, b ; q) .
\end{aligned}
$$

Then by (7.25) and the induction hypothesis on (7.20),

${ }_{556} P_{k}^{m+1}=c_{n+m, k} P_{k}^{m}+d_{n+m, k-1} P_{k-1}^{m}=\sum_{s=0}^{m+1} f_{n, k, k-s}^{m+1} P_{k-s}^{0}, \quad k=0, \ldots, n+m+1$, 
557

where

$$
f_{n, k, k-s}^{m+1}=c_{n+m, k} f_{n, k, k-s}^{m}+d_{n+m, k-1} f_{n, k-1, k-s}^{m}, \quad s=0, \ldots, m+1,
$$

and $f_{n, k, j}^{m}=0$ when $k=-1$ or $j \notin\{k-m, \ldots, k\}$.

To complete the proof, we need to verify the case $m+1$ of (7.21) and (7.22). First we verify (7.21). Let $\lambda=1 /(a b)$. By (7.26), (6.23), and the induction hypothesis on (7.21),

$$
\begin{aligned}
f_{n, k, k-s}^{m+1}= & \frac{\left(q^{n+m-k+1}, q^{n+m-k+1} \lambda ; q\right)_{1}}{\left(q^{n+m+1}, q^{n+m-2 k+1} \lambda ; q\right)_{1}} q^{s(n-k+s)}\left[\begin{array}{c}
m \\
s
\end{array}\right]_{q} \\
& \times \frac{\left(q^{k-s+1}, q^{-k} \lambda ; q\right)_{s}\left(q^{n+s-k+1}, q^{n+s-k+1} \lambda ; q\right)_{m-s}}{\left(q^{n+1} ; q\right)_{m}\left(q^{n-2 k+s} \lambda ; q\right)_{s}\left(q^{n-2 k+2 s+1} \lambda ; q\right)_{m-s}} \\
& +\frac{q^{n+m-k+1}\left(q^{k}, q^{-k} \lambda ; q\right)_{1}}{\left(q^{n+m+1}, q^{n+m-2 k+1} \lambda ; q\right)_{1}} \\
& \times q^{(s-1)(n-k+s)}[m \\
& \times \frac{\left(q^{k-s+1}, q^{-k+1} \lambda ; q\right)_{s-1}\left(q^{n+s-k+1}, q^{n+s-k+1} \lambda ; q\right)_{m-s+1}}{\left(q^{n+1} ; q\right)_{m}\left(q^{n-2 k+s+1} \lambda ; q\right)_{s-1}\left(q^{n-2 k+2 s+1} \lambda ; q\right)_{m-s+1}} \\
= & \frac{(q ; q)_{m}}{(q ; q)_{s}(q ; q)_{m+1}-s} \frac{q^{s(n-k+s)}}{\left(1-q^{n+m-2 k+1} \lambda\right)} \\
& \times \frac{\left(q^{k-s+1}, q^{-k} \lambda ; q\right)_{s}\left(q^{n+s-k+1}, q^{n+s-k+1} \lambda ; q\right)_{m+1-s}}{\left(q^{n+1} ; q\right)_{m+1}\left(q^{n-2 k+s} \lambda ; q\right)_{s}\left(q^{n-2 k+2 s+1} \lambda ; q\right)_{m+1-s}} Q(\lambda),
\end{aligned}
$$

where

$$
\begin{aligned}
Q(\lambda)= & \left(1-q^{m-s+1}\right)\left(1-q^{n+m-2 k+s+1} \lambda\right)+q^{m-s+1}\left(1-q^{s}\right)\left(1-q^{n-2 k+s} \lambda\right) \\
& =1+q^{n+2 m-2 k+2} \lambda-q^{m+1}-q^{n+m-2 k+1} \lambda \\
& =\left(1-q^{m+1}\right)\left(1-q^{n+m-2 k+1} \lambda\right) .
\end{aligned}
$$

Combining (7.27) and (7.28) yields (7.21) with $m$ replaced by $m+1$.

It remains to show that (7.22) holds for $m+1$. From (7.26), the induction hypothesis on (7.22), and (7.24), it follows that

$$
\sum_{s=0}^{m+1} f_{n, k, k-s}^{m+1}=\sum_{s=0}^{m+1}\left(c_{n+m, k} f_{n, k, k-s}^{m}+d_{n+m, k-1} f_{n, k-1, k-s}^{m}\right)
$$

$$
\begin{aligned}
& =c_{n+m, k} \sum_{s=0}^{m} f_{n, k, k-s}^{m}+d_{n+m, k-1} \sum_{s=1}^{m+1} f_{n, k-1, k-s}^{m} \\
& =c_{n+m, k}+d_{n+m, k-1}=1 .
\end{aligned}
$$


Another proof of Rogers' ${ }_{6} \phi_{5}$ sum (7.9) can be given by substituting (7.21) into (7.22), simplifying, and using M. Ismail's idea (see the discussion after (7.9)). The details are very similar to the proofs of Proposition 7.1 and Corollary 7.3.

Corollary 7.9 (Iterated Degree Elevation Formula) Let $\left\{f_{n, k, j}^{m}\right\}$ be defined by equation (7.21). Then

$$
\breve{B}_{j}^{n}(z ; a, b ; q)=\sum_{k=j}^{j+m} f_{n, k, j}^{m} \breve{B}_{k}^{n+m}(z ; a, b ; q), \quad j=0, \ldots, n .
$$

Proof Formula (7.29) follows from equation (7.20) in Proposition 7.8 applied to $\breve{P}(z)=\breve{B}_{j}^{n}(z ; a, b ; q)$, since in this case $P_{k}^{0}=\delta_{k, j}, k=0, \ldots, n$.

\section{References}

1. Andrews, G., Askey, R., Roy, R.: Special Functions, vol. 71. Cambridge University Press, Cambridge (1999)

2. Ait-Haddou, R., Sakane, Y., Nomura, T.: Chebyshev blossoming in Müntz spaces: toward shaping with Young diagrams. J. Comput. Appl. Math. 247, 172-208 (2013)

3. Gasper, G., Rahman, M.: Basic Hypergeometric Series, 2nd edn. Cambridge University Press, Cambridge (2004)

4. Goldman, R.: Pyramid Algorithms: A Dynamic Programming Approach to Curves and Surfaces for Geometric Modeling. Morgan Kaufmann Publishers, Burlington (2003)

5. Goldman, R.: An Integrated Introduction to Computer Graphics and Geometric Modeling. CRC Press, Taylor and Francis, New York (2009)

6. Goldman, R., Simeonov, P.: Quantum Bernstein bases and quantum Bézier curves. J. Comput. Appl. Math. 288, 284-303 (2015)

7. Goldman, R., Simeonov, P.: Two essential properties of $(q, h)$-Bernstein-Bézier curves. Appl. Numer. Math. 96, 82-93 (2015)

8. Goldman, R., Simeonov, P.: Generalized quantum splines. Comput. Aided Geom. Des. 47, 29-54 (2016)

9. Goodman, T.N.T., Mazure, M.-L.: Blossoming beyond extended Chebyshev spaces. J. Approx. Theory 109, 48-81 (2001)

10. Ismail, M.E.H.: Classical and Quantum Orthogonal Polynomials in One Variable, paperback edn., vol. 98. Cambridge University Press, Cambridge (2009)

11. Lyche, T.: Trigonometric splines; a survey with new results. In: Penã, J. (ed.) Shape Preserving Representations in Computer-aided Geometric Design, pp. 201-227. Nova Science Publishers Inc, New York (1999)

12. Mazure, M.-L.: Chebyshev spaces with polynomial blossoms. Adv. Comput. Math. 10, 219-238 (1999)

13. Mazure, M.-L.: Chebyshev spaces and Bernstein bases. Constr. Approx. 22, 347-363 (2005)

14. Pottmann, H.: The geometry of Tchebycheffian splines. Comput. Aided Geom. Des. 10, 181-210 (1993)

15. Koekoek, R., Lesky, P., Swarttouw, R.: Hypergeometric Orthogonal Polynomials and Their $q$ Analogues. Springer Monographs in Mathematics. Springer, Berlin (2010)

16. Ramshaw, L.: Blossoming: A Connect-the-Dots Approach to Splines, Digital Equipment Corp., Systems Research Center, Technical Report no. 19 (1987)

17. Ramshaw, L.: Bézier and B-splines as multiaffine maps. In: Earnshaw, R.A. (ed.) Theoretical Foundations of Computer Graphics and CAD, NATO ASI Series F, 40, pp. 757-776. Springer, New York (1988)

18. Ramshaw, L.: Blossoms are polar forms. Comput. Aided Geom. Des. 6, 323-358 (1989)

19. Saff, E.B., Snider, A.D.: Fundamentals of Complex Analysis for Mathematics, Science, and Engineering, 2nd edn. Prentice Hall, Upper Saddle River (1993)

20. Simeonov, P., Goldman, R.: Quantum B-splines. BIT 53, 193-223 (2013) 
21. Simeonov, P., Zafiris, V., Goldman, R.: $h$-Blossoming: a new approach to algorithms and identities for $h$-Bernstein bases and $h$-Bézier curves. Comput. Aided Geom. Des. 28, 549-565 (2011)

22. Simeonov, P., Zafiris, V., Goldman, R.: $q$-Blossoming: a new approach to algorithms and identities for $q$-Bernstein bases and $q$-Bézier curves. J. Approx. Theory 164, 77-104 (2012)

23. Stanley, R.: Enumerative Combinatorics, vol. 2. Cambridge University Press, Cambridge (1999) 